\title{
Ultrahigh-temperature film capacitors via homo/heterogeneous interfaces
}

\section{Ming Liu ( $\nabla$ m.liu@xjtu.edu.cn )}

xi'an jiaotong University

\section{Rui Lu}

xi'an jiaotong University

\section{Zhonghui Shen}

Wuhan University of Technology https://orcid.org/0000-0001-7828-0397

\section{Chunrui Ma}

xi'an jiaotong University

\section{Tingzhi Duan}

xi'an jiaotong University

Lu Lu

Xi'an Jiaotong University

Guangliang $\mathrm{Hu}$

xi'an jiaotong University

Tian-Yi Hu

xi'an jiaotong University

\section{Caiyin You}

Xi'an University of Technology

\section{Shaobo Mi}

xi'an jiaotong University

Chun-Lin Jia

xi'an jiaotong University

\section{Long-Qing Chen}

The Pennsylvania State University https://orcid.org/0000-0003-3359-3781

\section{Physical Sciences - Article}

Keywords: High-performance dielectric capacitors, energy storage, phase-field simulations

Posted Date: September 28th, 2021

DOl: https://doi.org/10.21203/rs.3.rs-926898/v1 
License: (c) (i) This work is licensed under a Creative Commons Attribution 4.0 International License. Read Full License 


\section{Ultrahigh-temperature film capacitors via homo/heterogeneous interfaces}

Rui $\mathrm{Lu}^{1,2 \dagger}$, Zhonghui Shen ${ }^{3,4 \dagger}$, Chunrui $\mathrm{Ma}^{2 \dagger^{* *}}$, Tingzhi Duan ${ }^{1,2}, \mathrm{Lu} \mathrm{Lu}^{1,2}$, Guangliang $\mathrm{Hu}^{1,2}$, Tian-Yi $\mathrm{Hu}^{2}$, Caiyin You $^{5}$, Shaobo Mi ${ }^{*}$, Chun-Lin $\mathrm{Jia}^{1,2,6}$, Long-Qing $\mathrm{Chen}^{7 *}$, Ming $\operatorname{Liu}^{1,2 *}$

1. School of Microelectronics, Xi'an Jiaotong University, Xi'an, China.

2. State Key Laboratory for Mechanical Behavior of Materials, Xi'an Jiaotong University, Xi'an, China.

3. State Key Laboratory of Advanced Technology for Materials Synthesis and Processing, Center of Smart Materials and Devices, Wuhan University of Technology, Wuhan, China.

4. International School of Materials Science and Engineering, Wuhan University of Technology, Wuhan, China.

5. School of Materials Science and Engineering, Xi'an University of Technology, Xi'an, China.

6. Ernst Ruska Centre for Microscopy and Spectroscopy with Electrons, ForschungszentrumJülich, D-52425, Jülich, Germany.

7. Department of Materials Science and Engineering, The Pennsylvania State University, University Park, Pennsylvania, United States.

$\dagger$ These authors contributed equally to this work.

*Corresponding author. Email: chunrui.ma@,xjtu.edu.cn (C. Ma), shaobo.mi@xjtu.edu.cn (S.-B. Mi), lqc3@psu.edu (L.-Q. Chen),

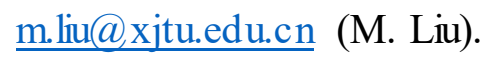




\begin{abstract}
High-performance dielectric capacitors are in high demand for advanced electronics and electric power systems. They possess high power density (on the order of Megawatt) and exhibit ultrafast charge/discharge capability (on a microsecond scale) and long-term storage lifetime ${ }^{1-5}$, and thus they are particularly demanded in pulse power systems such as high-power microwaves, hybrid electric vehicles, and highfrequency inverters. However, their relatively low operating temperature limits their widespread applications ${ }^{6-9}$. Here, guided by phase-field simulations, we synthesized capacitors with an energy storage density of 55.4 joules per cubic centimeter, energy efficiency of over $82 \%$, and superior thermal stability and fatigue properties at record high operating temperature of $400^{\circ} \mathrm{C}$. These ultrahigh-temperature performances are achieved through a relatively simple method of introduction and engineering of interfaces within the capacitors, which greatly improve their high-temperature stability, relaxation behavior, and breakdown strength. Our work not only successfully fabricated capacitors with potential applications in high-temperature electric power systems and electronic technologies but also opens up a promising and general route for designing high-performance electrostatic capacitors through interface engineering.
\end{abstract}




\section{Main text}

Dielectric capacitors belong to one important family of energy storage elements used in every electronic equipment. However, their relatively low operating temperature greatly limits their applications in harsh environment, for example underground oil and gas exploration (beyond $\left.200^{\circ} \mathrm{C}\right)^{6}$. Even though a cooling system can be integrated in some applications, for example, in hybrid electric vehicles to reduce the working temperature to below $105^{\circ} \mathrm{C}$ for the capacitors made of the commercially biaxially oriented polypropylene $(\mathrm{BOPP})^{6-9}$, the auxiliary cooling loop undoubtedly adds extra weight, volume, as well as energy consumption. Therefore, increasing the operating temperature of dielectric capacitors while maintaining high energy storage density is extremely critical to realizing their new technological applications.

To possess high energy storage density $\left(U_{\mathrm{e}}\right)$ and high efficiency $(\eta)^{10}$ and to be able to operate at high temperatures, a dielectric capacitor must satisfy the following requirements: (i) stable high permittivity with low loss in the applied temperature range $^{6,11}$; (ii) low electric conductivity at elevated temperature to minimize the leakage current of the dielectric and ensure a high breakdown strength $^{5,9,12}$; (iii) high thermal conductivity to allow efficient heat dissipation ${ }^{5}, 13,14$. There have been extensive efforts to develop dielectric thin films to meet these requirements ${ }^{15-20}$. For example, lead-based dielectrics are first considered due to their high Curie temperature, and thus high and stable permittivity over a wide temperature range, and an upper operation temperature of up to $280^{\circ} \mathrm{C}$ with energy density $\sim 26 \mathrm{~J} / \mathrm{cm}^{3}$ were achieved ${ }^{21}$. Due to the environmental concerns associated with lead-based dielectrics, a number of approaches 
have been attempted to develop lead-free dielectric materials for potential applications in high-temperature dielectric capacitors, including ion substitutions ${ }^{15,22,23}$, composited dielectrics $^{3,16,24}$, and the artificially configured structures combining relaxor ferroelectric, antiferroelectric, ferroelectric and linear dielectric ${ }^{25-28}$. The highest operation temperature in lead-free materials with an energy storage density of $\sim 30 \mathrm{~J} / \mathrm{cm}^{3}$ is $250^{\circ} \mathrm{C}^{20}$, which is still far below many application requirements. Therefore, the main objective of this work is to explore a novel interfacial engineering approach to boost the upper limit of working temperature and energy storage density of lead-free dielectric materials to realize high-temperature applications of dielectric capacitors.

We first conducted phase-field simulations (see Methods for Phase-field Simulations) to investigate the dielectric breakdown path, local polarization distribution, and heat flux of dielectrics with artificially configured interfaces. In particular, we consider four representative dielectric structures (Fig. 1a): pure matrix (PM), vertical interfaces (VI), parallel interfaces (PI), and regular interlayer interfaces (II). Our hypothesis is that the interfaces in the dielectric may alter the dielectric breakdown path and thus enhance the dielectric breakdown strength. Indeed, more tortuous and shorter broken paths (black region) occur in the dielectrics with parallel interfaces and regular interlayer interfaces (Fig. 1b). 


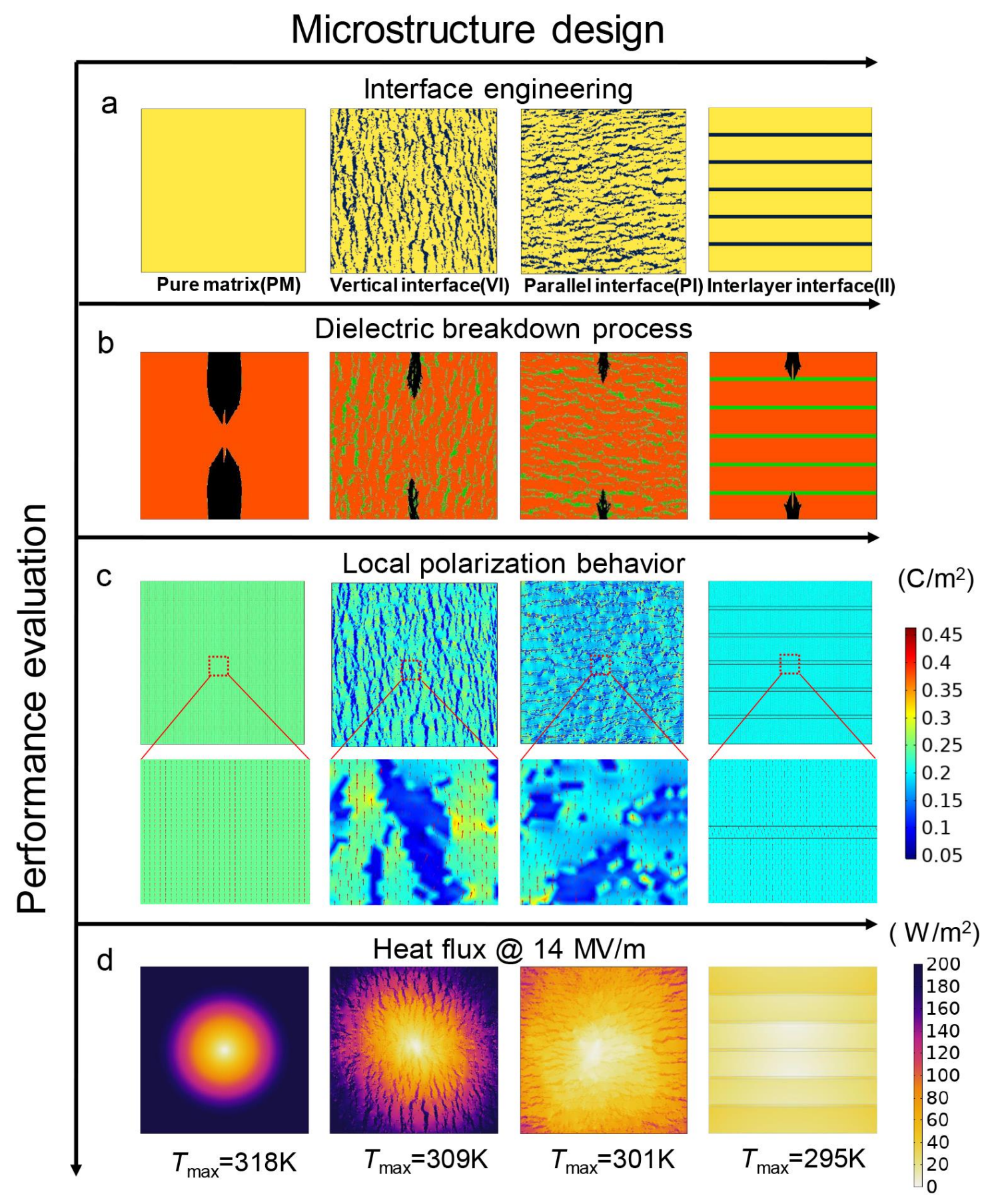

Fig. 1| Design of new microstructure for enhancement of energy performances via interface

engineering. a, Schematics of the microstructure in phase-field models based on different interface systems: pure matrix, (PM), vertical interfaces (VI), parallel interfaces (PI) and interlayer interfaces (II) b, Breakdown phase evolution. c, Local polarization distribution. d, Heat flux and corresponding maximal temperature when operating at $14 \mathrm{MV} / \mathrm{m}$. 
The interfaces in PI, VI and II break the long-range ordered domains into random nanodomains (Fig. 1c), which is beneficial to domain switching and reducing the remnant polarization, and hence increasing the charge-discharge efficiency. We also studied the thermal stability by modeling the heat dissipation (heat flux in Fig. 1d) for a dielectric operating at $14 \mathrm{MV} / \mathrm{m}$ with a surrounding temperature of $293 \mathrm{~K}$. It is found that introducing the homo/heterogeneous interfaces into dielectrics could lower the maximal temperature from $318 \mathrm{~K}$ in $\mathrm{PM}$ to $295 \mathrm{~K}$ in II since the interfaces reduce the Joule heating and act as the exit for thermal runway. Therefore, the interfaces could synergistically enhance the energy storage performance and high-temperature stability.

Guided by the simulation results on the benefits of introducing interfaces into dielectrics in reducing thermal effect, improving the voltage endurance, and modulating the domain morphology, we attempt to synthesize a composition-gradient multilayer structure, namely heterogeneous interface (HEI) to mimic a regular interlayer interface in a dielectric, and PI or VI interface (referring to as homogeneous interface (HOI)) in a single-phase dielectric by tuning the growth conditions. The integrated homogeneous/heterogeneous interfaces will take advantage of each other's merit to achieve high-performance energy storage at high-temperatures. $\mathrm{BaHf}_{x} \mathrm{Ti}_{1-x} \mathrm{O}_{3}$ (BHTO), as one of the members of $\mathrm{BaTiO}_{3}$ based dielectrics, exhibits stable permittivity and low dielectric loss in a wide temperature range. Thus, it is selected to construct a series of interface configuration including VI, PI and II demonstrated in Fig. 2a.

The BHTO films were fabricated on (001) Nb-doped $\mathrm{SrTiO}_{3}(\mathrm{Nb}: \mathrm{STO})$ substrates by the radio frequency magnetron sputtering system. The epitaxial growth of the films 
was verified by X-ray diffraction (Supplementary Fig. 1). The columnar structure with VI and PI is achieved in the 17 at.\% Hf-doped $\mathrm{BaTiO}_{3}$ (BHTO-17) films by controlling film-growth conditions, especially the growth temperature. The density of VI and PI increases as the growth temperature decreases from $850^{\circ} \mathrm{C}$ (Fig. 2b) to $700^{\circ} \mathrm{C}($ Fig. $2 \mathrm{c})$.

a
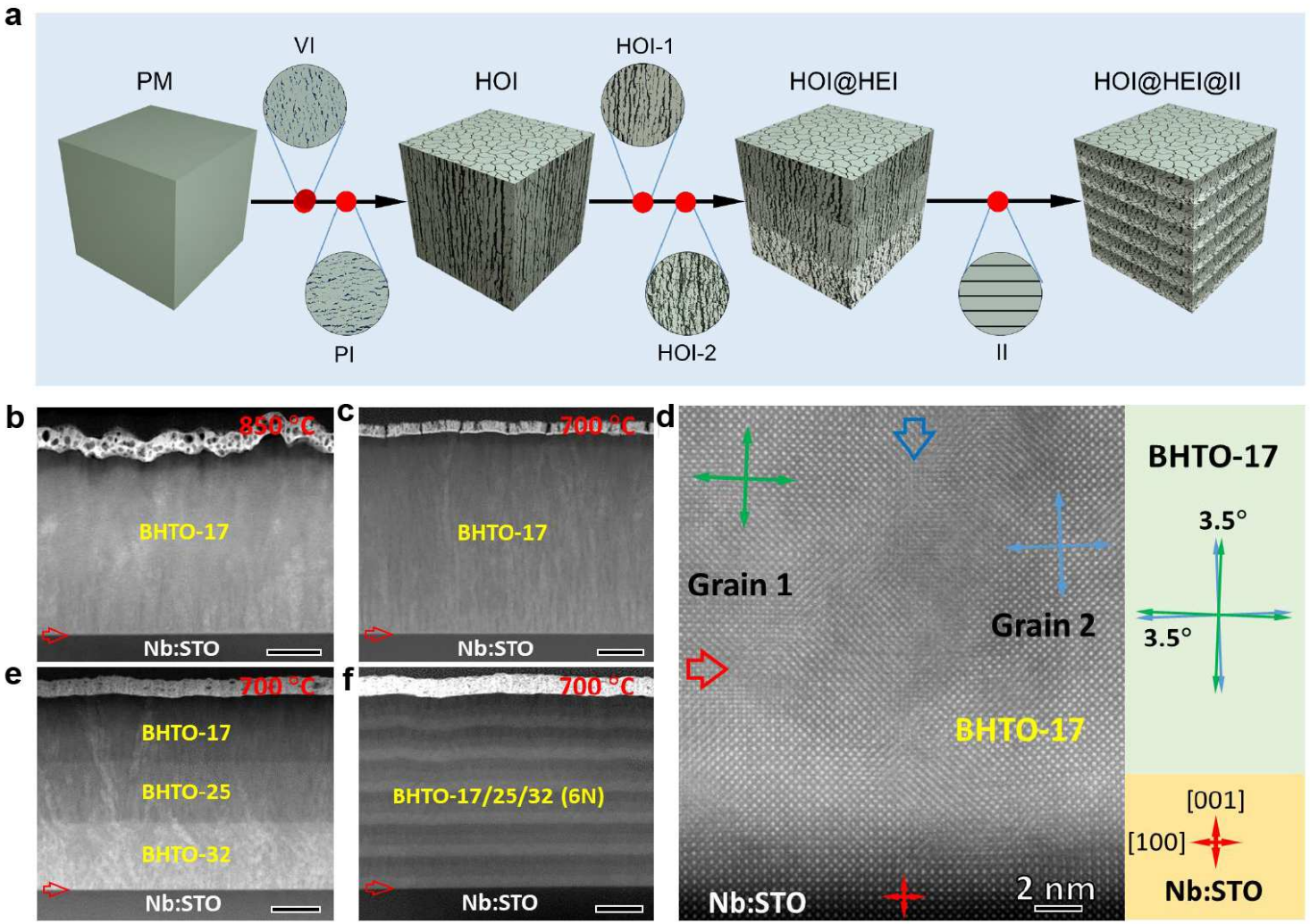

BHTO-17

Fig. 2| Fabrication and microstructure characterization of the interface-

engineered film sys tems.a, Schematic of the synthesis route of VI, PI and II. (b and c) HAADF-

STEM images of BHTO-17 films grown on (001) Nb-doped $\mathrm{SrTiO}_{3}(\mathrm{Nb}: \mathrm{STO})$ substrates at $850^{\circ} \mathrm{C}$ (corresponding to PM structure) and $700^{\circ} \mathrm{C}$ (corresponding to PI and VI structure), respectively. d, A typical HAADF-STEM image of BHTO-17 films containing VI and PI, indicated by a vertical blue and a horizontal red arrow, respectively. The in-plane and out-of-plane rotation between the adjacent grains is indicated by double-head arrows, which is illustrated in the insert. e, HAADFSTEM image of a tri-layer film system containing BHTO-32, BHTO-25 and BHTO-17 layers. f, HAADF-STEM image of the tri-layer film in $\mathbf{f}$ with six growth cycles. HAADF-STEM images in (b-f) are viewed along the [010] direction of the $\mathrm{Nb}$ :STO substrate, and the film-substrate interface is indicated by a horizontal red arrow. Scale bar in $(\mathbf{b}, \mathbf{c}, \mathbf{e}$ and $\mathbf{f})$ is $100 \mathrm{~nm}$. 
A lower growth temperature reduces grain growth leading to nanograin structures. As demonstrated in reciprocal space mappings (RSMs) (Supplementary Fig. 2) and a high-angle annular dark field (HAADF) scanning transmission electron microscopy (STEM) image of BHTO-17 films prepared at $700^{\circ} \mathrm{C}$ (Fig. 2d), the formation of VI and PI results from grain boundary migration and coalescence as demonstrated in Fig. 2 d. Uniform elemental distribution in the BHTO films from energy-dispersive X-ray spectroscopy (EDS) mapping (Supplementary Fig. 3) indicates negligible elemental segregation. In order to build the structure as depicted in Fig. 2a, we selected 25 and 32 at.\% Hf-doping in the $\mathrm{BaTiO}_{3}$ films with the composition, lattice parameter, and permittivity close to BHTO-17 to construct a tri-layer film denoted as HOI@HEI (Fig. 2e). The contrast variation is clearly visible under the HAADF-STEM imaging conditions $^{29}$, indicating the appearance of the HEI in the film. Note that the VI and PI still exist in each BHTO layer. Of course, the density of the II within the film system can be manipulated by controlling the number of HOI@HEI film-growth cycles $(\mathrm{N})$. For example, compared with the film fabricated with one growth cycle (Fig. 2e), the density of the interface increases by a factor of 6 (Fig. $2 \mathrm{f}$ ) in the film fabricated with six growth cycles $(6 \mathrm{~N})$. Thus, the patterned structure including VI, PI, and II is integrated into the BHTO films successfully without changing the overall thickness of the film system.

As expected by the phase field simulations, the built interfaces can significantly enhance $E_{\mathrm{b}}$ evaluated using a two-parameter Weibull distribution fitting (Fig. 3a). The $E_{\mathrm{b}}$ values are $7.4 \mathrm{MV} / \mathrm{cm}$ for $\mathrm{HOI}$ film and $8.8 \mathrm{MV} / \mathrm{cm}$ for the HOI@HEI film, which 
are $23 \%$ and $47 \%$ higher than that for the $\mathrm{PM}$ film $(6.0 \mathrm{MV} / \mathrm{cm})$, respectively. The combination of HOI, HEI and II (HOI@HEI@II) results in a maximum $E_{\mathrm{b}}=9.6$ $\mathrm{MV} / \mathrm{cm}$, which is $60 \%$ higher than that of the PM film, and $>1100 \%$ higher than that of the pure BTO films $(\sim 0.79 \mathrm{MV} / \mathrm{cm})^{30}$. It is worth mentioning that the $E_{\mathrm{b}}(9.6 \mathrm{MV} / \mathrm{cm})$ obtained through the interface design is the highest breakdown field strength among all previous reports ${ }^{31-35}$. The Weibull modulus $\beta$ that describes the scatter of breakdown field data also gradually increases from 15 (the PM film) to 33 (HOI@HEI@II film) upon introduction of interfaces into the films. The relatively large $\beta$ in HOI@HEI@II film suggests a narrowed distribution of $E_{\mathrm{b}}$ values and an improvement of dielectric reliability. The suppressed electric breakdown stems from the greatly reduced leakage current by two orders of magnitude from $3.5 \times 10^{-5}$ to $6.7 \times 10^{-7} \mathrm{~A} / \mathrm{cm}^{2}$ at a DC field of $4 \mathrm{MV} / \mathrm{cm}$ (Fig. 3b). The combination of homogeneous and heterogeneous interfaces effectively prolongs the ohmic conduction to $2.37 \mathrm{MV} / \mathrm{cm}$, and delays the on-set of the Fowler-Nordheim tunneling mechanism (Supplementary Fig. 4). Besides the increase in $E_{\mathrm{b}}$, the $P_{\mathrm{m}} / \mathrm{P}_{\mathrm{r}}$ value is another key parameter for determining the energy storage performance of a dielectric. We extracted the values of $P_{\mathrm{m}}$ and $\mathrm{P}_{\mathrm{r}}$ and plotted $P_{\mathrm{m}} / \mathrm{P}_{\mathrm{r}}$ in Fig. 3c. It is found the introduced interfaces indeed can break down the large domains into nano-domains, thus enhancing the dipole switching and relaxation behavior (Supplementary Fig. 5). The films with designed interfaces clearly outperform the PM film in terms of $U_{\mathrm{e}}$ and $\eta$ (Fig. 3d) evaluated through their unipolar $P-E$ loops (Supplementary Fig. 6). Strikingly, the $U_{\text {e }}$ reaches a maximum value of $131 \mathrm{~J} / \mathrm{cm}^{3}$ under 9.2 MV/cm for the film with HOI@HEI@II, which is 2.5 times higher than the $51 \mathrm{~J} / \mathrm{cm}^{3}$ 
measured in the PM film. The $\eta$ value is improved from 65 to $86 \%$ at the same $E_{b}$ of 5.0 MV/cm with increasing interface density. More importantly, the HOI@HEI@II film maintains a high $\eta$ of $\sim 80 \%$ even at $E_{b}=9.2 \mathrm{MV} / \mathrm{cm}$ approaching the breakdown electric fields.

a

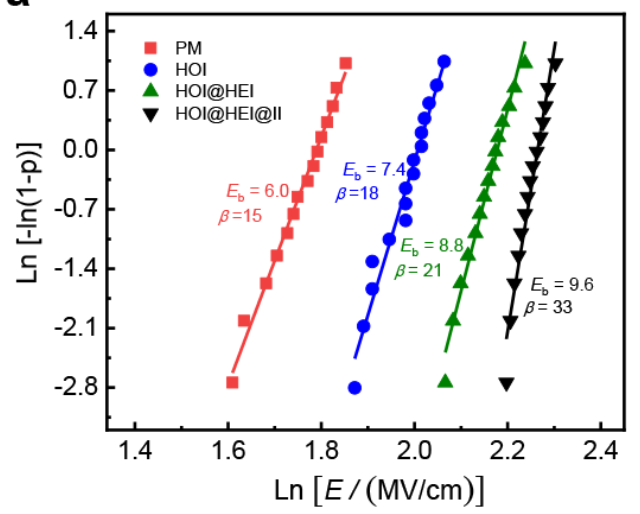

C

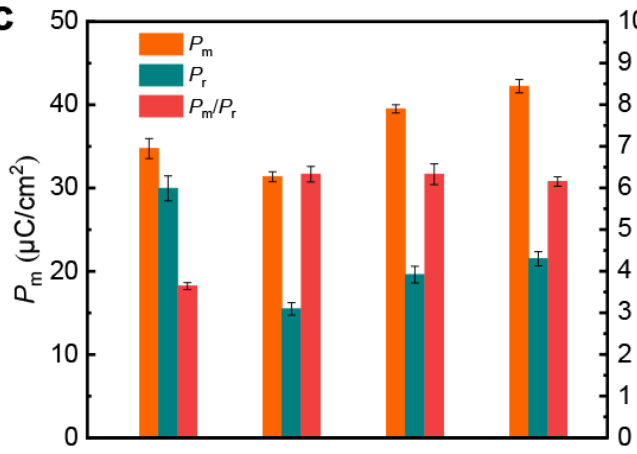

PM HOI HOI@HEI HOI@HEI@II b
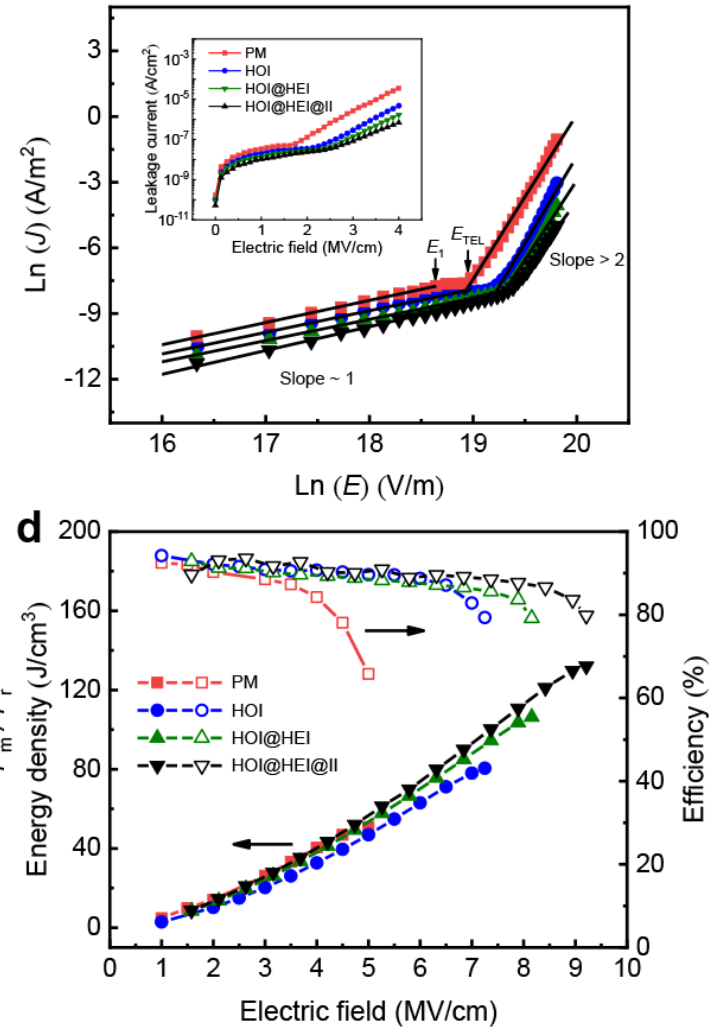

Fig. 3| Break down strengths and energy storage performances of the BHTO films with

different interface configurations. a, The characteristic breakdown strength $E_{\mathrm{b}}$ (unit: $\mathrm{MV} / \mathrm{cm}$ ) and

Weibull parameter $\beta$ are derived from the BHTO films for different interface configurations. $\mathbf{b}$,

Logarithmic plot of leakage current density versus electric field for the films. c, Comparison of the energy storage performances of the films at their breakdown fields. The $P_{\mathrm{m}}, P_{\mathrm{r}}$ and $P_{\mathrm{m}} / P_{\mathrm{r}}$ are determined from ten points of the PM,HOI,HOI@HEI and HOI@HEI@II samples. The error bars in $\mathbf{c}$ present the standard deviation of the corresponding data. d, Energy density and efficiency values of the films with respect to applied electric fields up to their breakdown fields. 
To obtain the temperature dependence of the dielectric performances of the structures, we obtained $P-E$ loops at different temperatures (Supplementary Fig. 7) and the temperature dependent energy density $U_{\mathrm{e}}$ and efficiency $\eta$ (Fig. 4a). The first observation is the broadening of operating temperature and the increase in energy density with the introduction of interfaces. The operating temperature extends from $250^{\circ} \mathrm{C}$ to $400^{\circ} \mathrm{C}$ as the film structure changes from PM to HOI@HEI@II structure, and the corresponding energy storage density can be as high as $55.4 \mathrm{~J} / \mathrm{cm}^{3}$ over the temperature range $-100^{\circ} \mathrm{C}$ to $400^{\circ} \mathrm{C}$. To our best knowledge, there have been no reports of operating temperatures over $300^{\circ} \mathrm{C}$ for dielectric materials. We found that the energy storage density is much higher than the benchmark value $\left(26.8 \mathrm{~J} / \mathrm{cm}^{3}\right)$ obtained at $280^{\circ} \mathrm{C}^{21}$. It should be pointed out that the excellent temperature stability covers most of the intended applications (i.e., South Pole $-90^{\circ} \mathrm{C}$ to the engineering working temperature in aerospace $250^{\circ} \mathrm{C}$, or geotherm $320^{\circ} \mathrm{C}$ ). The energy density $U_{\mathrm{e}}$ of the HOI@HEI@II structure may reach $72.8 \mathrm{~J} / \mathrm{cm}^{3}$ under the upper temperature at $300^{\circ} \mathrm{C}$ (Supplementary Fig. 8). In addition, both $U_{\mathrm{e}}$ and $\eta$ exhibit outstanding thermal stability over such a wide temperature range with $\eta$ maintaining above $82 \%$, in contrast to PM which shows a sharp decrease at $250^{\circ} \mathrm{C}$. We attribute the broad working temperature range and stable energy storage properties to a number of factors. First, the maximum permittivity temperature $\left(T_{\mathrm{m}}\right)$ shifts downwards with increasing interface density (Fig. 4b) owing to the increased relaxor diffuseness factor $\gamma$ defined as the degree of diffuseness which varies between 1 (normal ferroelectrics) and 2 (ideal relaxor ferroelectrics). The $\gamma$ increases from 1.36 for the PM film to2.00 for the HOI@HEI@II 
film in the measurement temperature range from room temperature to $500^{\circ} \mathrm{C}$ derived from modified Curie-Weiss law (Supplementary Fig. 9). The increased relaxor feature behavior of the HOI@HEI@II structure is responsible for its maximum and most stable permittivity. Second, the lowest dielectric and reduced conduction losses (Supplementary Fig. 10) effectively decrease Joule heating and temperature rise inside the dielectric (Fig. 1d), thus retarding thermal breakdown.

a
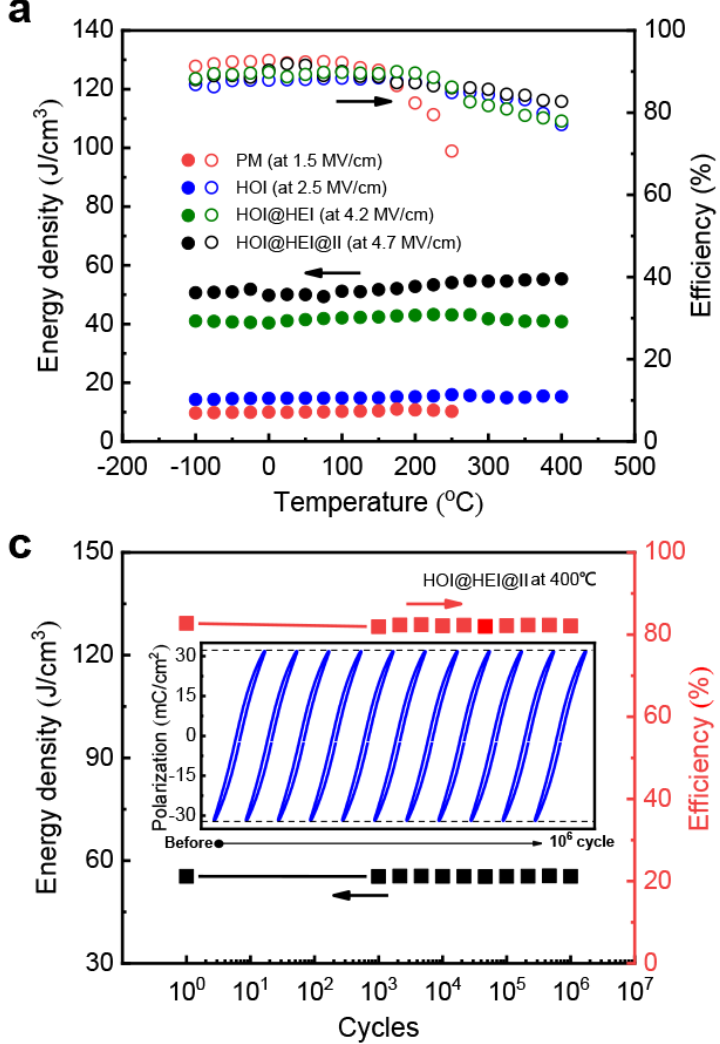

b
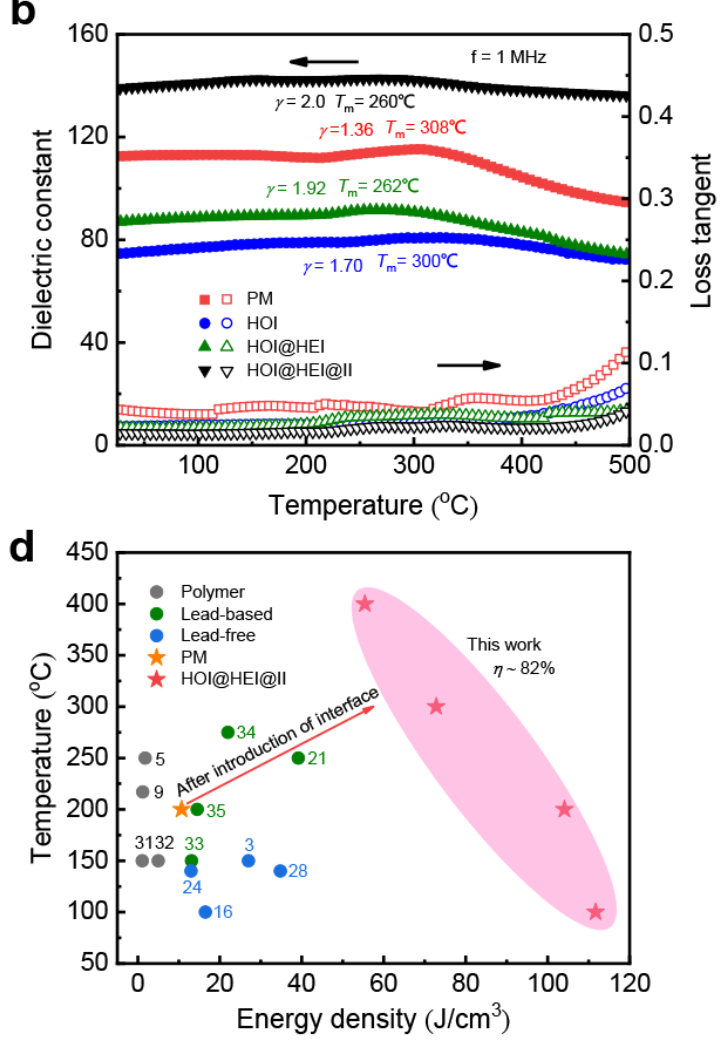

Fig. 4| Dielectric, reliability and stability evaluations of the BHTO film with different interface

configurations. a, Temperature-dependent energy storage performances of the films at different electric fields. b, Temperature-dependent dielectric permittivity and loss tangent of the films at frequency of $1 \mathrm{MHz}$. c, Energy storage performances of the film at electric field of $4.7 \mathrm{MV} / \mathrm{cm}$ with respect to charging-discharging cycling numbers. $\mathbf{d}$, The comparison diagram of the energy storage performances at elevated temperature for the representative films materials. 
Finally, the introduced interfaces reduce leakage current (Fig. 3b), extend the ohmic conduction behavior (Supplementary Fig. 4), and greatly suppress the leakage current at high temperatures (Supplementary Fig. 11), which suppresses electron avalanche and thus Joule heating. Performance stability and reliability are another critical prerequisite for the commercialization of dielectric capacitors. The fatigue behaviors of the HOI@HEI@II film are tested under 4.7 MV/cm at 400 $\mathrm{C}$ (Fig. 4c). We found the variation in $U_{\mathrm{e}}$ and $\eta$ less than $2 \%$ after $1 \times 10^{6}$ charge-discharge cycles, which is close to the commercial standard. Additionally, the films with designed interfaces can quickly discharge the stored energy in $0.45 \mu \mathrm{s}$, and the power density is stable over the test temperature range and can be as high as $105 \mathrm{MW} / \mathrm{cm}^{3}$ at $400^{\circ} \mathrm{C}$ (Supplementary Fig. 12). These excellent performances are superior to previously reported polymer thin films $5,9,31,32$, lead-based thin films ${ }^{21,33-35}$, and lead-free thin films $^{3}, 16,24,28$ (Fig. 4d). They meet the requirements for applications in almost all the extreme conditions.

The designed homo/heterogeneous interfaces in a composition gradient system not only broadens the working temperature to $400^{\circ} \mathrm{C}$ but also improves the energy storage density and efficiency. The findings in this work should have broad impacts on energy storage devices for harsh environments. This interface-design strategy can be applied to other material systems, such as $0.85 \mathrm{BaTiO}_{3}-0.15 \mathrm{Bi}\left(\mathrm{Mg}_{0.5} \mathrm{Zr}_{0.5}\right) \mathrm{O}_{3}, \mathrm{BZT}$, and PLZT to improve their overall energy storage performances. It should also be generally applicable to any insulating systems in which the homo/heterogeneous interfaces can be manipulated for electric device applications at ultra-high temperatures. 



\section{References}

1. Li, J. L., Li, F., Xu, Z. \& Zhang, S. J. Multilayer lead-free ceramic capacitors with ultrahigh energy density and efficiency. Adv. Mater.30, 1802155 (2018).

2. Kim, J. et al. Ultrahigh capacitive energy density in ion-bombarded relaxor ferroelectric films. Science. 369, 81-84 (2020).

3. Pan, H. et al. Ultrahigh-energy density lead-free dielectric films via polymorphic nanodomain design. Science. 365, 578-582 (2019).

4. Chu, B. J. et al. A dielectric polymer with high electric energy density and fast discharge speed. Science. 313, 334-336 (2006).

5. Li, Q. et al. Flexible high-temperature dielectric materials from polymer nanocomposites. Nature. 523, 576-579(2015).

6. Li, Q. et al. High-temperature dielectric materials for electrical energy storage. Annu. Rev. Mater. Res. 48, 219-243 (2018).

7. Rabuffi, M. \& Picci, G. Status quo and future prospects for metallized polypropylene energy storage capacitors. IEEE. T. Plasma. Sci. 30, 1939-1942 (2002).

8. Jiang, J. Y. et al. Polymer nanocomposites with interpenetrating gradient structure exhibiting ultrahigh discharge efficiency and energy density. Adv. Energy. Mater. 9, 1803411 (2019).

9. Azizi, A. et al. High-performance polymers sandwiched with chemical vapor deposited hexagonal boron nitrides as scalable high-temperature dielectric materials. Adv. Mater. 29, 1701864 (2017). 
10. Cheng, H. B. et al. Demonstration of ultra-high recyclable energy densities in domain-engineered ferroelectric films. Nat. Commun. 8, 1999 (2017).

11. Hu, G. L. et al. Enhanced energy density with a wide thermal stability in epitaxial

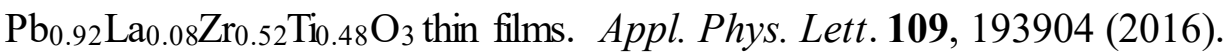

12. Yuan, C. et al. Polymer/molecular semiconductor all-organic composites for hightemperature dielectric energy storage. Nat. Commun. 11, 3919 (2020).

13. Shen, Z. H. et al. Phase-field model of electrothermal breakdown in flexible hightemperature nanocomposites under extreme conditions. Adv. Energy. Mater. 8, 1800509 (2018).

14. Shen, Z. H. et al. Phase-field modeling and machine learning of electric-thermalmechanical breakdown of polymer-based dielectrics. Nat. Commun. 10, 1843 (2019).

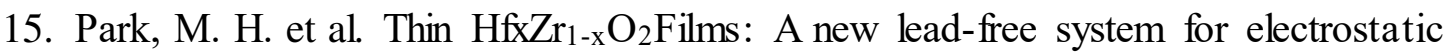
supercapacitors with large energy storage density and robust thermal stability. $A d v$. Energy. Mater. 4, 1400610 (2014).

16. Pan, H. et al. Giant energy density and high efficiency achieved in bismuth ferritebased film capacitors via domain engineering. Nat. Commun.9, 1813 (2018).

17. Luo, N. N. et al. Constructing phase boundary in $\mathrm{AgNbO}_{3}$ antiferroelectrics: pathway simultaneously achieving high energy density and efficiency. Nat. Commun. 11, 4824 (2020).

18. Pan, Z. B. et al. Fatigue-free aurivillius phase ferroelectric thin films with ultrahigh energy storage performance. Adv. Energy. Mater. 10, 2001536 (2020). 
19. Zhou, Y. et al. High-throughput, and environmentally benign approach to polymer dielectrics exhibiting significantly improved capacitive performance at high temperatures. Adv. Mater.30, 1805672 (2018).

20. Hu, T. Y. et al. Enhanced energy storage performance of lead-free capacitors in an ultrawide temperature range via engineering paraferroelectric and relaxor ferroelectric multilayer films. ACS Appl. Mater. Interfaces. 12, 25930-25937 (2020).

21. Liu, C. et al. Energy storage and polarization switching kinetics of (001)-oriented $\mathrm{Pb}_{0.97} \mathrm{La}_{0.02}\left(\mathrm{Zr}_{0.95} \mathrm{Ti}_{0.05}\right) \mathrm{O}_{3}$ antiferroelectric thick films. Appl. Phys. Lett. 108, 112903 (2016).

22. Zhao, Y., X. Hao, H. \& Li, M. L. Dielectric properties and energy-storage performance of $\left(\mathrm{Na}_{0.5} \mathrm{Bi}_{0.5}\right) \mathrm{TiO}_{3}$ thick films. J. Alloy. Compd. 601, 112-115 (2014).

23. Liang, Z. S. et al. High-performance $\mathrm{BaZr}_{0.35} \mathrm{Ti}_{0.65} \mathrm{O}_{3}$ thin film capacitors with ultrahigh energy storage density and excellent thermal stability. J. Mater. Chem. A. 6, 12291-12297 (2018).

24. Pan, $\mathrm{H}$. et al. $\mathrm{BiFeO}_{3}-\mathrm{SrTiO}_{3}$ thin film as a new lead-free relaxor-ferroelectric capacitor with ultrahigh energy storage performance. J. Mater. Chem. A . 5, 59205926 (2017).

25. Silva, J. P. B. et al. High-performance ferroelectric-dielectric multilayered thin films for energy storage capacitors. Adv Funct Mater. 29, 1807196 (2018).

26. Fan, Q. L. et al. Significantly enhanced energy storage density with superior thermal stability by optimizing $\mathrm{Ba}\left(\mathrm{Zr}_{0.15} \mathrm{Ti}_{0.85}\right) \mathrm{O}_{3} / \mathrm{Ba}\left(\mathrm{Zr}_{0.35} \mathrm{Ti}_{0.65}\right) \mathrm{O}_{3}$ multilayer 
structure. Nano Energy. 51, 539-545 (2018).

27. Chen, J. Y., Tang, Z. H., Yang, B. \& Zhao, S. F. Ultra-high energy storage performances regulated by depletion region engineering sensitive to the electric field in PNP-type relaxor ferroelectric heterostructural films. J. Mater. Chem.A.8, 8010-8019(2020).

28. Sun, Z. X. et al. Ultrahigh energy storage performance of lead-free oxide multilayer film capacitors via interface engineering. Adv. Mater. 29, 1604427 (2017).

29. Nellist, P. D. \& Pennycook, S. J. The principles and interpretation of annular darkfield Z-contrast imaging. Adv Imag Elect Phys. 113, 147-203 (2000).

30. Yang, B. B. et al. Energy storage properties in $\mathrm{BaTiO}_{3}-\mathrm{Bi}_{3}{ }_{25} \mathrm{La}_{0.75} \mathrm{Ti}_{3} \mathrm{O}_{12}$ thin films. Appl. Phys. Lett. 113, 033904 (2018).

31. Li, Q. et al. Sandwich-structured polymer nanocomposites with high energy density and great charge-discharge efficiency at elevated temperatures. Proc Natl Acad Sci U S A. 113, 9995-10000 (2016).

32. Li, H. et al. Scalable polymer nanocomposites with record high-temperature capacitive performance enabled by rationally designed nanostructured inorganic fillers. Adv Mater. 31, 1900875 (2019).

33. Zhao, Y., Hao, X. H. \& Zhang, Q. Energy-storage properties and electrocaloric effect of $\mathrm{Pb}_{(1-3 \mathrm{x} / 2)} \mathrm{La}_{\mathrm{x}} \mathrm{Zr}_{0.85} \mathrm{Ti}_{0.15} \mathrm{O}_{3}$ antiferroelectric thick films. ACS Appl. Mater. Interfaces. 6, 11633-11639 (2014).

34. Liu, C. et al. Energy storage and polarization switching kinetics of (001)-oriented $\mathrm{Pb}_{0.97} \mathrm{La}_{0.02}\left(\mathrm{Zr}_{0.95} \mathrm{Ti}_{0.05}\right) \mathrm{O}_{3}$ antiferroelectric thick films. Appl. Phys. Lett. 108, 
112903 (2016).

35. Nguyen, M. D., Houwman, E. P., Do, M. T. \& Rijnders, G. Relaxor-ferroelectric thin film heterostructure with large imprint for high energy-storage performance at low operating voltage. Energy. Storage. Materials. 25, 193-201 (2020). 


\section{Data availability}

The data that support the findings of this study are available from the corresponding authors upon reasonable request.

\section{Code availability}

The LabView codes used in this work are available from the corresponding authors upon reasonable request.

\section{Acknowledgements :}

M.L., C.M., G.H. and C.J. were supported by the Natural Science Foundation of China (grant numbers 52172235, U2032168, 62001371, 51702255, and 51390472), National “973" projects of China (grant numbers 2015CB654903) and the Fundamental Research Funds for the Central Universities. Z.S. was supported by the Natural Science Foundation of China (grant numbers 52002300) and The Major Research Plan of NSFC (grant numbers 92066103). C.Y. was supported by the Natural Science Foundation of China (grant numbers 51771145) and the ISF-NSFC joint research program (grant numbers 51961145305).

Author contributions: M.L. and L.-Q.C. supervised the work. M.L. and C.R.M. conceived the idea and designed the experiments. R.L. carried out the multilayer thin film fabrication and characterizations. C.R.M. and T.Z.D. carried out the singlelayer thin film fabrication and characterizations. T.Y.H and G.L.H. conducted the FORC measurements and analyzed the data. R.L., C.R.M. and M.L. analyzed the data. Z.H.S. and L.-Q.C. performed the phase-field simulations. L.L., C.-L. J. and S.-B.M. 
performed the STEM measurements. R.L., Z.-H.S., S.-B.M. and M.L. wrote the first draft of the manuscript. All authors discussed the results and edited the manuscript. Competing interests: The authors declare no competing interests.

Data and materials availability: The authors declare no competing interests.

Additional information

Supplementary Materials

Correspondence and requests for materials should be addressed to C.R.M.,

S.B.M., L.-Q.C. or M.L. 


\section{Supplementary Files}

This is a list of supplementary files associated with this preprint. Click to download.

- Supplementaryinformation.pdf 